\title{
Dynamics in binary cluster crystals
}

\author{
Manuel Camargo ${ }^{1,2}$, Angel J Moreno ${ }^{3}$, and Christos N Likos ${ }^{4,1}$ \\ ${ }^{1}$ Institute of Theoretical Physics, Heinrich Heine University of Düsseldorf, \\ Universitätsstraße 1, D-40225 Düsseldorf, Germany \\ ${ }^{2}$ Dirección Nacional de Investigaciones, Universidad Antonio Nariño, Kra 3 Este \\ 47a-15 Bogotá, Colombia \\ ${ }^{3}$ Centro de Física de Materiales (CSIC,UPV/EHU) and Materials Physics Center \\ MPC, Paseo Manuel de Lardizabal 5, E-20018 San Sebastián, Spain \\ ${ }^{4}$ Faculty of Physics, University of Vienna, Boltzmanngasse 5, A-1090 Vienna, Austria \\ E-mail: camargo@thphy.uni-duesseldorf.de, wabmosea@ehu.es, and \\ christos.likos@univie.ac.at
}

\begin{abstract}
As a result of the application of coarse-graining procedures to describe complex fluids, the study of systems consisting of particles interacting through bounded, repulsive pair potentials has become of increasing interest in the last years. A well known example is the so-called Generalized Exponential Model (GEM-m), for which the interaction between particles is described by the potential $v(r)=$ $\epsilon \exp \left[-(r / \sigma)^{m}\right]$. Interactions with $m>2$ lead to the formation of a novel phase of soft matter consisting of cluster crystals. Recent studies on the phase behavior of binary mixtures of GEM- $m$ particles have provided evidence for the formation of novel kinds of alloys, depending on the cross interactions between the two species. This work aims to study the dynamic behavior of such binary mixtures by means of extensive molecular dynamics simulations, and in particular to investigate the effect of the addition of non-clustering particles on the dynamic scenario of one-component cluster crystals. Analogies and differences with the one-component case are revealed and discussed by analyzing self- and collective dynamic correlators.
\end{abstract}

PACS numbers: 61.20.Ja, 64.70.Pf, 82.70.-y

Keywords: soft matter, diffusion, cluster crystal, hopping 


\section{Introduction}

Coarse-graining procedures provide a general way to represent mesoscopic aggregates of several architectures (e.g., linear and hyperbranched polymers, micelles, microgels, etc.) as particles interacting via an effective, isotropic potential $V_{\text {eff }}(r)$. When the center of mass of such aggregates can coincide without violating excluded volume interactions, $V_{\text {eff }}(r)$ turns out to be ultrasoft and bounded [1, 2, 3]. These characteristics may lead to cluster formation providing $V_{\text {eff }}(r)$ belongs to the so-called $Q^{ \pm}$-class, i.e. its Fourier transform $(\mathrm{FT}) \hat{V}_{\text {eff }}(q)$ displays an oscillatory decay around zero (potentials with positive FT are referred as $Q^{+}$potentials) [4, 5]. Recent numerical investigations of amphiphilic dendrimers and of ring polymers with different degree of knotedness demonstrate that such macromolecules are candidates for experimental realizations of the former clustering scenario, since their effective interactions are indeed of the $Q^{ \pm}$-class [6, 7, 8]. A particular theoretical realization of $Q^{ \pm}$potentials is given by the generalized exponential model (GEM-m), for which the interaction is described by the potential

$$
v(r)=\epsilon \exp \left[-\left(\frac{r}{\sigma}\right)^{m}\right] \quad(m>2),
$$

where $r$ is the interparticle distance, $\sigma$ is a measure of the particle size, and $\epsilon$ is the interaction strength. As density increases, the GEM- $m$ system shows, for $m>2$, a firstorder transition from a fluid of clusters to a cluster bcc-crystal above the triple point temperature, and a subsequent structural phase transition from a cluster bcc to a cluster fcc crystal. This occurs at arbitrarily high temperatures [9, 10], contrary to the case of $Q^{+}$-systems, which exhibit reentrant crystallization in the temperature-density plane [11. A remarkable feature of the cluster fcc crystals is that upon increasing the density the lattice constant approaches a constant value $a_{\mathrm{fcc}}=2 \pi \sqrt{3} / q^{*}$, with $q^{*}$ the wave vector at which $\hat{v}(q)$ takes its absolute minimum. An obvious consequence of the densityindependent value of $a_{\mathrm{fcc}}$ is that the population of each lattice site scales proportionally with density. These properties are rather different from usual crystallization features

in colloidal systems. Moreover, these differences are not restricted to the structural properties but also to the dynamical ones. Regarding the slow dynamics in such cluster crystals, incessant hopping between clusters has been revealed, which fully changes the initial identity of the clusters without altering the lattice structure. Another peculiar dynamic feature of these systems is a decoupling between self- and collective out-oflattice correlations [12, 13].

Recently, the phase behavior of mixtures containing a non-clustering component $(m=2)$ and a clustering component $(m=4)$ has been investigated within the framework of the Density Functional Theory (DFT) [14, 15]. For the case of non-demixing systems, evidence was found for the formation of novel kinds of alloys, i.e. mixed cluster crystals [15. Though static features of these mixtures have been studied in detail, to the best of our knowledge no information has been reported on the corresponding dynamical aspects. This article aims to shed light on such aspects. By means of extensive molecular dynamics (MD) simulations, the findings displayed by one-component cluster crystals 
[12, 13] are extended to mixtures of clustering and non-clustering particles. Analogies and differences with the one-component case are revealed and discussed by analyzing self- and collective dynamic correlators. The article is organized as follows: Model and simulation details are given in Section 2. Static and dynamic features are presented and discussed in Section 3. Conclusions are given in Section 4.

\section{Model and Simulation}

As a previous step, the stability limits of the homogeneous fluid phase (coexistence and $\lambda$ lines) were determined for representative examples of the former class of binary mixtures. These limits were obtained by solving the Ornstein-Zernike (OZ) equation within the mean field approximation (MFA) [16]. Based on such results, the specific systems to be simulated were determined and thus the parameters defining the mixture (relative particle size, mixture compositions, temperature and density, self- and crossinteractions) were chosen in such a way as to avoid the region of demixing in the phase diagram. Because of the high total densities to be considered, the former parameters were also selected with the goal of reducing computational expense.

The investigated system was a mixture of big GEM-8 particles and small GEM-2 (gaussian) particles. This mixture contains a non-clustering component (GEM-2, in the following referred as $A$-particles) and a cluster-forming component (GEM-8, in the following referred as $B$-particles). The dynamic features of this system were investigated at fixed temperature $T$ for a broad range of densities $\rho=\rho_{A}+\rho_{B}$ and several relative compositions $x=\rho_{B} / \rho$. The former densities are defined as $\rho_{\alpha}=N_{\alpha} / V, N_{\alpha}$ being the number of particles of the species $\alpha$ and $V$ the total volume. In what follows, we choose units in which Boltzmann's constant $k_{\mathrm{B}}$ has the value $k_{\mathrm{B}}=1$. The cross-interaction was chosen to be a GEM-4 potential, which also displays cluster formation. In this way, the following interaction parameters were adopted for the potential (1): $\epsilon_{\alpha \beta}=\epsilon=1.0$, $\sigma_{A A}=0.3 \sigma, \sigma_{A B}=0.6 \sigma, \sigma_{B B}=\sigma=1.0$, and $m_{A A}=2, m_{A B}=4, m_{B B}=8$. With these parameters, a common cutoff range $R_{c}=1.5 \sigma$ was introduced in the simulation for all the interactions.

MD simulations were performed in a cubic box with periodic boundary conditions. The equations of motion were integrated in the velocity Verlet scheme [17, with a time step $\Delta t / \tau$ ranging from 0.001 to 0.005 , where $\tau=\sqrt{m \sigma^{2} / \epsilon}$ and a common mass $m=1$ was used for all the particles. The size of the simulation box, $L_{\text {box }}$, was typically of 7 or 8 times the lattice constant $a_{\mathrm{fcc}}$ of the considered mixture. The value of $a_{\mathrm{fcc}}$ was obtained from DFT as explained in [14, 15]. In the following, density, time, distance, and wave vector will be given, respectively, in units of $\sigma^{-3}, \tau, \sigma$, and $\sigma^{-1}$. Initial configurations were generated by placing the particles uniformly at the sites of the fcc lattice defined by $a_{\mathrm{fcc}}$. An equilibration run was performed in which the system was thermalized at temperature $T=0.30$ by periodic velocity rescaling. Typical equilibration times ranged from $2 \times 10^{5}$ to $2 \times 10^{6}$ time steps according to the studied density. After reaching equilibrium, manifested by the absence of any drift in internal energy and pressure, a 

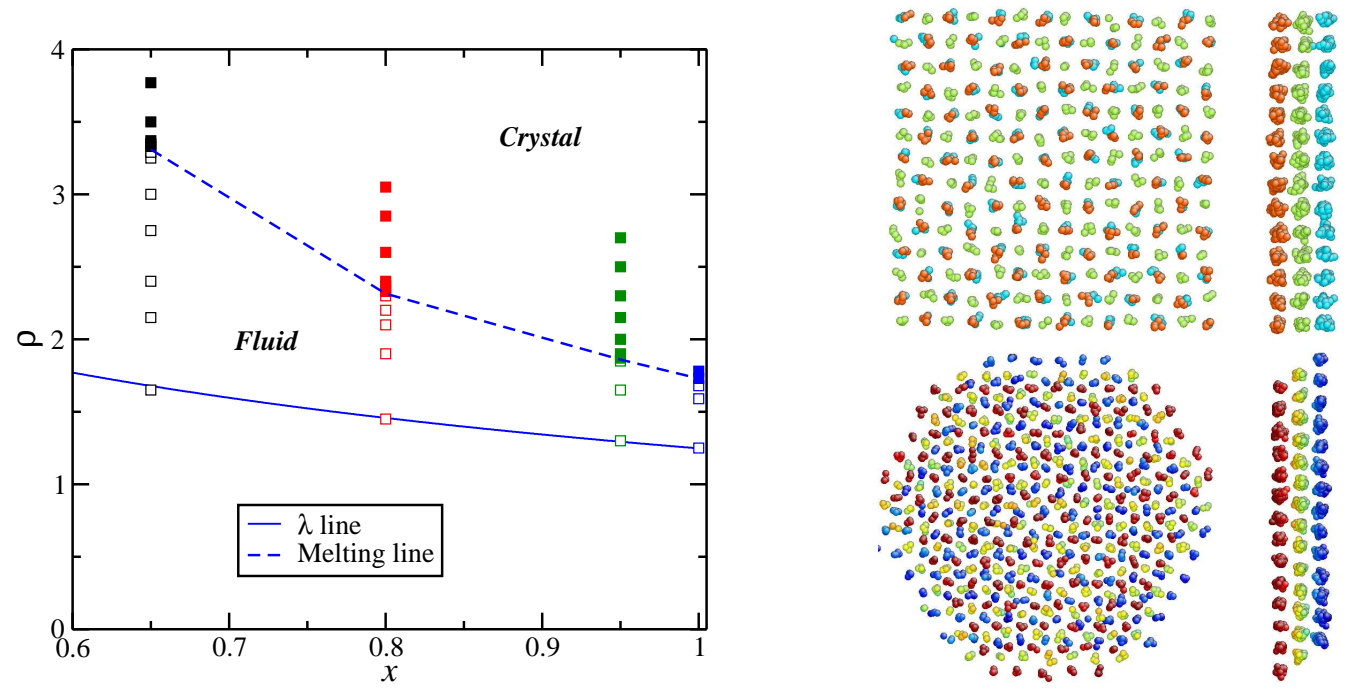

Figure 1. Left: A segment, at the high- $x$ /moderate $\rho$ region, of the phase diagram of the studied ultrasoft mixture. Empty and filled symbols indicate the simulated state points in the $(\rho, x)$-plane, at both sides of the melting line. The latter is displayed as a dashed guideline for the eyes. It is estimated from the emergence of Bragg peaks in the partial static structure factors (see below). The solid line is the $\lambda$-line, obtained from the OZ-MFA equations. Right: Slabs of the simulation box for $x=0.65$ and $\rho=3.50$, parallel to the (001)-plane (top) and to the (111)-plane (bottom). For the sake of clarity, only GEM-8 particles are displayed, in both frontal and lateral views, with a size much smaller than the real $\sigma$. Particles in different layers are represented with different colors.

production run was performed in the microcanonical ensemble, at different densities, for compositions $x=0.65,0.80$ and 0.95 . Typical production runs ranged from $10^{6}$ to $10^{8}$ time steps.

\section{Results and Discussion}

\subsection{Phase diagram and static structure}

The partial structure factors $S_{\alpha \beta}(q)(\alpha, \beta \in\{A, B\})$ are related to the propensity of a fluid to sustain spontaneous density fluctuations of wave vector $q$. They are defined as

$$
S_{\alpha \beta}(q)=\frac{\left\langle\rho_{\alpha}(\mathbf{q}, 0) \rho_{\beta}(-\mathbf{q}, 0)\right\rangle}{\sqrt{N_{\alpha} N_{\beta}}}
$$

with $\rho_{\alpha}(\mathbf{q}, t)=\sum_{j=1}^{N_{\alpha}} \exp \left[i \mathbf{q} \cdot \mathbf{r}_{j}^{\alpha}(t)\right]$ and the sum is performed over the coordinates $\mathbf{r}_{j}^{\alpha}$ of all particles belonging to the species $\alpha \pm$ According to the OZ equations they take,

$\ddagger$ For uniform phases, the statistical average $\langle\cdots\rangle$ in the right hand side of eq. (2) above renders its left hand side a function of $q=|\mathbf{q}|$ only. For the crystalline phases to be considered in what follows, we have performed an additional rotational average over symmetry-related values of the reciprocal vectors, so that the resulting quantities (static and time-dependent correlation functions) are shown as functions of $q$ only. 
Table 1. For each investigated composition $x$, density $\rho^{*}$ for the $\lambda$-line, parameters defining the fcc lattice $\left(D\left(q^{*}\right)=0, a_{\mathrm{fcc}}=2 \pi \sqrt{3} / q^{*}\right.$, see [14] $)$ and box size $L_{\mathrm{box}}$ used in the MD.

\begin{tabular}{lllll}
\hline$x$ & $\rho^{*}$ & $q^{*}$ & $a_{\mathrm{fcc}}$ & $L_{\mathrm{box}}$ \\
\hline 0.65 & 1.68 & 5.64 & 1.9296 & 13.5070 \\
0.80 & 1.46 & 5.74 & 1.8960 & 15.1677 \\
0.95 & 1.29 & 5.84 & 1.8635 & 14.9079 \\
1.00 & 1.25 & 5.86 & 1.8571 & 14.8571 \\
\hline
\end{tabular}

in the homogeneous phase, the generic form $S_{\alpha \beta}(q)=N_{\alpha \beta}(q) / D(q)$ where, within the MFA, the denominator depends on the Fourier transform of the interaction potentials according to

$$
D(q)=1+\frac{\rho}{T} \hat{U}_{s}(q)+\left(\frac{\rho}{T}\right)^{2} x(1-x) \hat{U}_{d}(q)
$$

where $\hat{U}_{s}(q)=(1-x) \hat{v}_{A A}(q)+x \hat{v}_{B B}(q)$ and $\hat{U}_{d}(q)=\hat{v}_{A B}(q) \hat{v}_{B B}(q)-\hat{v}_{A B}^{2}(q)$ [14]. Due to their generic form, the partial structure factors will diverge for all those conditions where $D(q)=0$, and therefore the homogeneous fluid will be unstable. If the divergence takes place at $q=0$, demixing occurs and the spinodal line denotes the locus of state points where $D(0)=0$. On the other hand, a divergence at some $q^{*}>0$ signals an instability in the fluid (which is referred as Kirkwood instability) with respect to a periodic modulation of the density. The locus of state points where this instability takes place corresponds to the so-called $\lambda$-line [15].

For the system at hand, the last scenario is indeed the case, where the instability dominating the phase behavior is mainly due to the $Q^{ \pm}$-nature of the species $B$. Figure 1 displays in the $(x, \rho)$-plane the corresponding $\lambda$-line estimated from MFA. Since this line is related to the limit of stability for the homogeneous fluid phase, it is expected that close to it the system will tend to crystallize into solids whose lattice constant will be dictated by $q^{*}$ (see Table 11). By visual inspection of the configuration snapshots, state points for which the initial crystal structure was stable were discriminated from those for which the latter melted. The melting line estimated in this way was consistent with the analysis of Bragg peaks in the partial structure factors (see below). No transition to other crystalline structures was observed during the simulations, confirming the fcc structure as the underlying lattice in the investigated crystalline states. The typical snapshots of Figure 1 indeed exhibit the expected ABC staking of the (111)-planes. It should be noted that the apparent contradiction of having, in Figure1, a fluid phase that is stable beyond the $\lambda$-line is an artefact arising from applying the MFA in calculating the latter. The MFA becomes increasingly accurate at $T$ grows, whereas we are working here at $T=0.3$. Nevertheless, this has no consequences in what follows, since we are employing the MFA $\lambda$-line merely as an indicator of the region in which alloy formation (crystallization) is expected, without basing any further quantitative predictions on its precise location. 

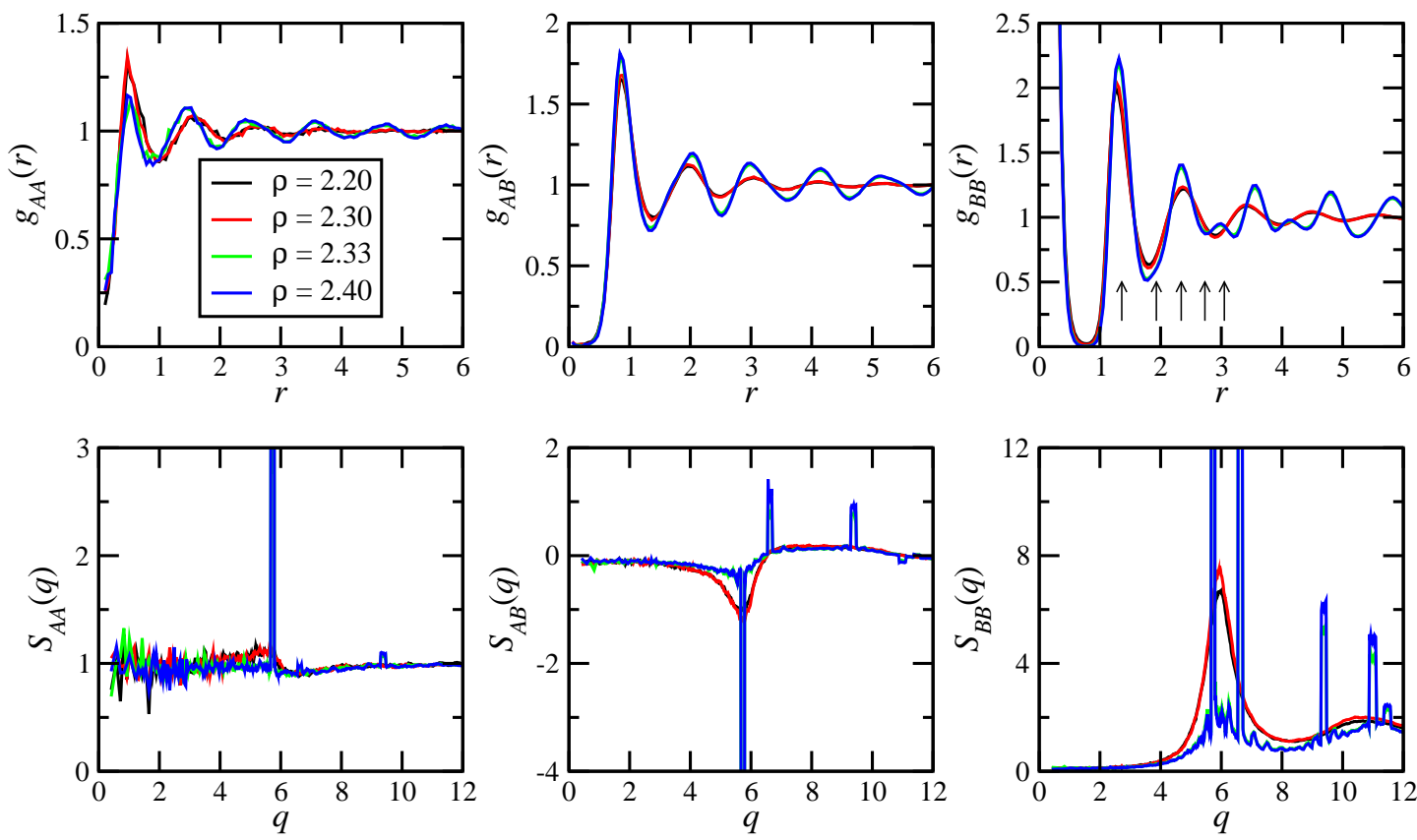

Figure 2. Radial distribution functions (top) and structure factors (bottom) for $x=0.80$ and different densities. The arrows in the plot of $g_{B B}(r)$ indicate the position of the $k$ th-neighbor in a fcc lattice, i.e. $d_{k}=\sqrt{k} d_{\mathrm{nn}}=\sqrt{k / 2} a_{\mathrm{fcc}}$. The values of the selected densities are the same in all panels (see legend for $g_{A A}(r)$ ).

More detailed structural information can be gained by considering the partial radial distribution functions $g_{\alpha \beta}(r)$, which measure the probability to find a pair of particles, of species $\alpha$ and $\beta$, at a mutual distance $r$. In figure 2 these quantities are displayed for $x=0.80$ and different densities. As expected, $g_{B B}(r)$ exhibits a large peak at $r=0$ (namely $g_{B B}(r \rightarrow 0) \sim 30$ ), indicating the formation of clusters, whose size extends up to $d_{\mathrm{c}} \sim 0.75$. The second peak at $r \sim 1.34$ corresponds to the nearestneighbor distance in the fcc crystal, $d_{\mathrm{nn}}=a_{\mathrm{fcc}} / \sqrt{2}$. The radial distribution function for distinct pairs, $g_{A B}(r)$, displays rather different features. The correlation hole at $r \rightarrow 0$ indicates that the small $A$-particles avoid the lattice sites and tend to localize in the interstitials between $B$-clusters. Concerning correlations between pairs of $A$-particles, $g_{A A}(r)$ displays finite small values $(\sim 0.2)$ at $r \rightarrow 0$. This means that for the considered conditions, no appreciable clustering of the species $A$ is induced by the clustering of the species $B$.

On increasing the density, a significant structural change occurs in the system, which is evidenced by the emergence of well-defined peaks of all $g_{\alpha \beta}(r)$ at large distances (see top panels of figure 21). This change is better reflected in the corresponding partial structure factors $S_{\alpha \beta}(q)$. As shown in bottom panels for the largest densities, the emergence of Bragg peaks in $S_{B B}(q)$ indicates a rather ordered structure, which still retains some characteristics of the fluid phase. On the other hand, the nearly flat structure of $S_{A A}(q)$ clearly suggests the presence of a, nearly ideal, fluid phase of 
$A$-particles. This fluid phase is immersed in the crystalline matrix of clusters of $B$ particles. Strictly speaking, the $A$-particles form a very weakly modulated fluid, so that the mixture of localized $B$-particles and delocalized $A$-particles forms a sublattice melt phase. The one-particle density of the $A$-particles, however, cannot be strictly uniform, since the crystallized $B$-particles act on the $A$-species as a periodically modulated external potential. This structural scenario has its dynamic counterpart, as will be shown below.

The occupation number $n$ of a given lattice site, i.e., the number of $B$-particles in the associated cluster, is obviously an integer number. The average occupation is in general non-integer, as a result of (at least two) significant contributions of distinct values of $n$ all over the lattice. For example, in the crystalline system of composition $x=0.80$ and density $\rho=2.40$ around the $65 \%$ and $25 \%$ of the clusters are composed respectively by three and four $B$-particles. This percentages were estimated by counting the coordination number of each particle for $r<d_{\mathrm{c}} \sim 0.75$. On passing, it was also estimated by using the same criterion that around $90 \%$ of the $A$-particles are isolated from particles of the same species. The former results reflect that the lattice is full of defects which might be expected to break its stability. As discussed in Ref. [13] for the one-component cluster crystal, the stability of the lattice is actually maintained by incessant hopping of the $B$-particles between distinct clusters. This is also the case in the mixtures considered in the present work, as discussed in the next subsection.

\subsection{Dynamics in real space}

A first step to gain some insight into the transport properties of the system is provided by the mean squared displacement $\left\langle\Delta r_{\alpha}^{2}(t)\right\rangle$ (MSD). This quantity is shown in figure 3 for different densities and relative compositions $x=0.65$ and $x=0.95$. In the case of the $A$-particles a rapid crossover is observed, for all the investigated densities, between short-time ballistic motion $\left(\left\langle\Delta r_{\alpha}^{2}(t)\right\rangle \propto t^{2}\right)$ and long-time diffusion $\left(\left\langle\Delta r_{\alpha}^{2}(t)\right\rangle \propto t\right)$. This is the usual dynamic behavior of a highly mobile fluid phase. Thus, as anticipated by the static correlations (see above), the mixture reaches a phase in which the species $A$ shows a fluid-like behavior in a crystalline matrix of clusters of $B$-particles.

The MSD of the $B$-particles shows a similar simple behavior in the fluid phase. However, an abrupt dynamic change is revealed by crossing the crystallization point. Thus, an intermediate plateau regime arises between the ballistic and diffusive limits, extending over longer time intervals as density increases. This regime is associated

with the temporary trapping of the $B$-particles within the clusters [12, 13]. The large oscillations at the beginning of the plateau regime are the signature of strong intracluster vibrational motion. Such short-time strong oscillations are an artifact of the simulated Newtonian dynamics. They are expected to be strongly damped under more realistic Brownian dynamics (BD); it has been shown that they even vanish in Monte Carlo (MC) simulations [18]. Finally, at long times, the MSD displays a crossover to diffusive behavior. This scenario, which has been previously observed in the cluster crystal phase 

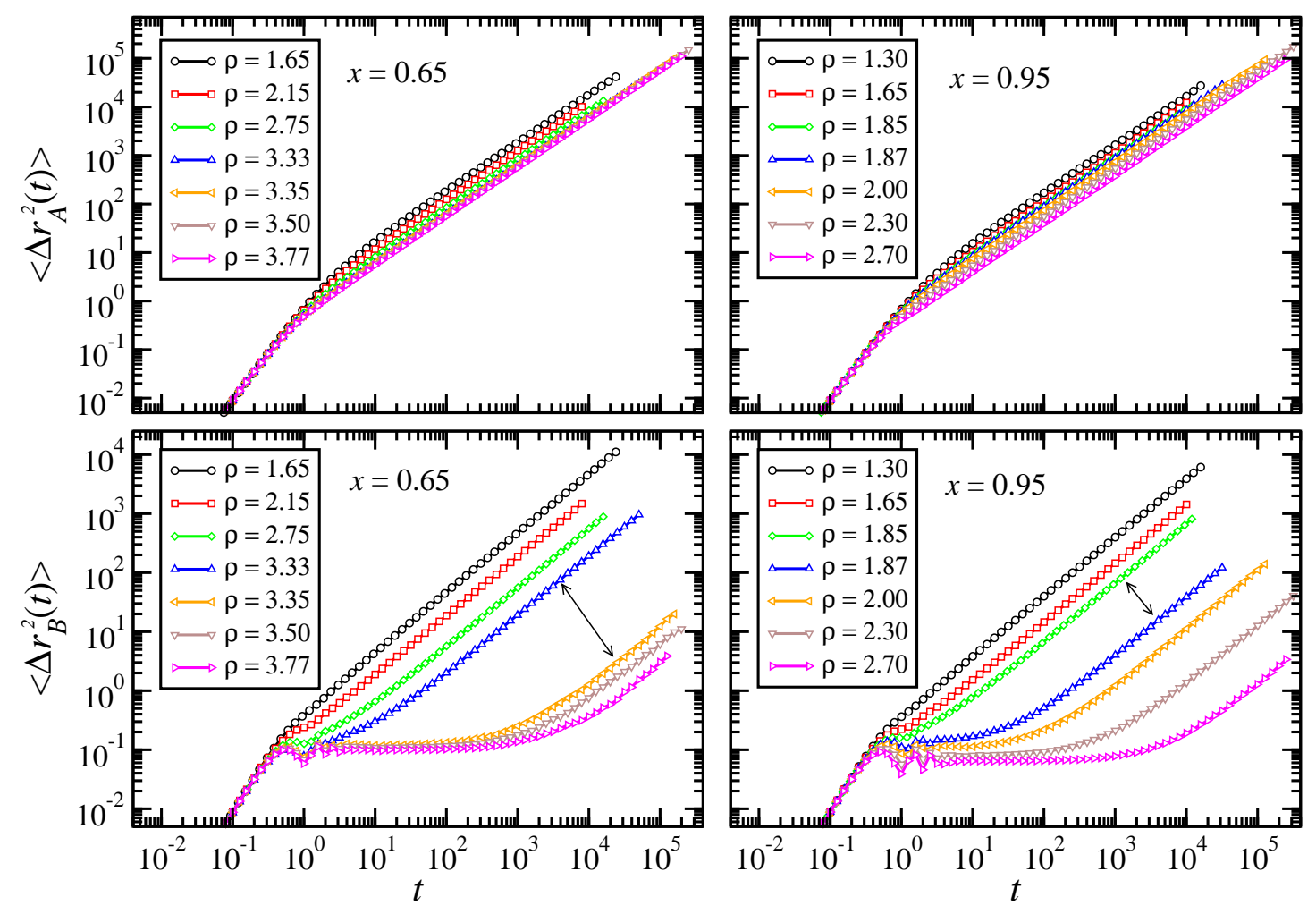

Figure 3. MSD of both species for $x=0.65$ (left panels) and $x=0.95$ (right panels). The arrows in bottom panels separate fluid states for the $B$-particles from crystal clusters (see also Figure 1).

of the pure GEM-8 system [12, 13], does not originate from the contribution to the MSD of rare events involving a few diffusing $B$-particles. As anticipated above, it is the result of incessant hopping of $B$-particles between neighboring lattice sites. Thus, the hopping mechanism fully changes the initial identity of each cluster without altering the overall crystalline lattice structure [12, 13].

This hopping mechanism is clearly reflected in the van Hove self-correlation, $G_{\alpha}^{(\mathrm{s})}(r, t)$, of the $B$-particles. Figure 4 shows a representative example for composition $x=0.80$ and density $\rho=3.05$. A sequence of well-defined peaks is present in $G_{B}^{(s)}(r, t)$, corresponding to different distances between lattice sites. With increasing time, the first peak in $G_{B}^{(\mathrm{s})}(r, t)$ decreases while peaks located at larger distances grow progressively, corresponding to particles moving away from their original home clusters. By simple integration of $G_{B}^{(s)}(r, t)$ from its first minimum to $r \rightarrow \infty$, it is found that only the $30 \%$ of the $B$-particles are located at $t \sim 50000$ in their initial home cluster. As expected from the observations in the MSD (see above) the van Hove self-function for the nonclustering species $A$ displays a more simple behavior. For $t \sim 50$ the initial peak at $r \approx 0.3$ has already vanished. At that time scale almost all the $A$-particles have left their initial interstitial positions and $G_{A}^{(\mathrm{s})}(r, t)$ exhibits simple Gaussian behavior.

We can estimate the diffusivity of each species from the relation $D_{\alpha}=$ 

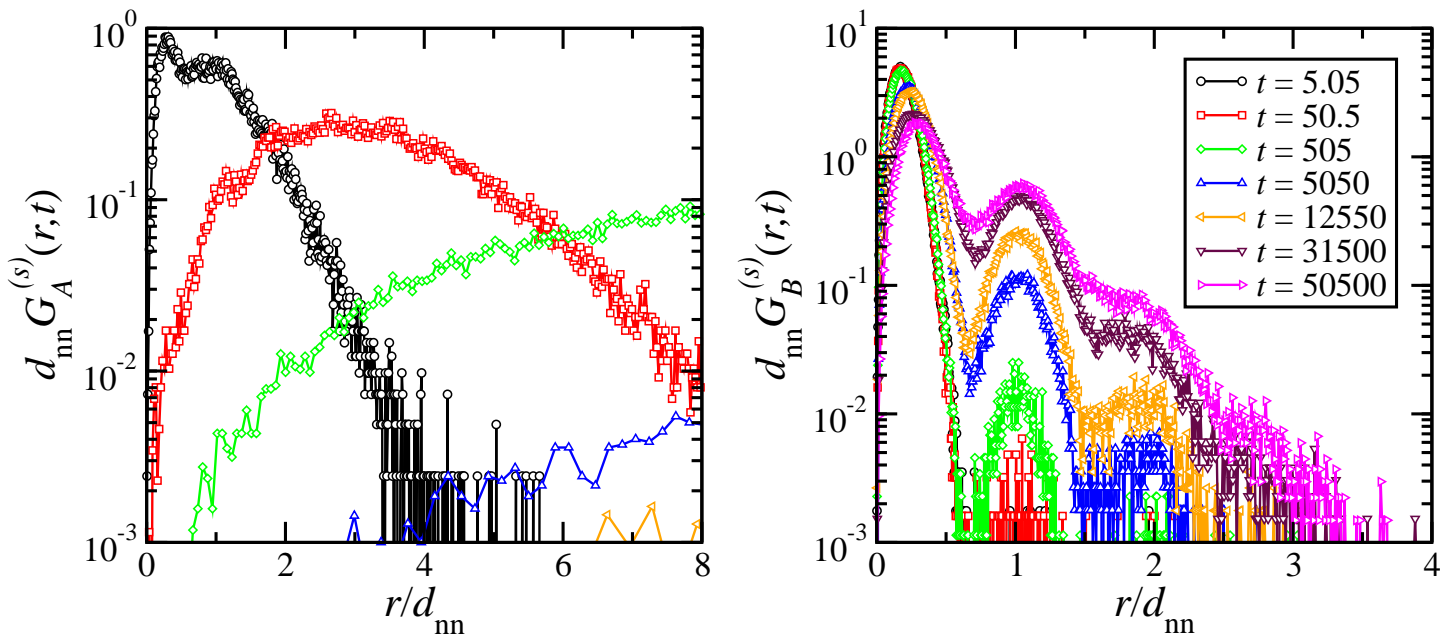

Figure 4. Van Hove self-correlation function at different times for $x=0.80$ and $\rho=3.05$ (left: $A$-particles, right: $B$-particles). Distances are rescaled by $d_{\mathrm{nn}}$ (see text).

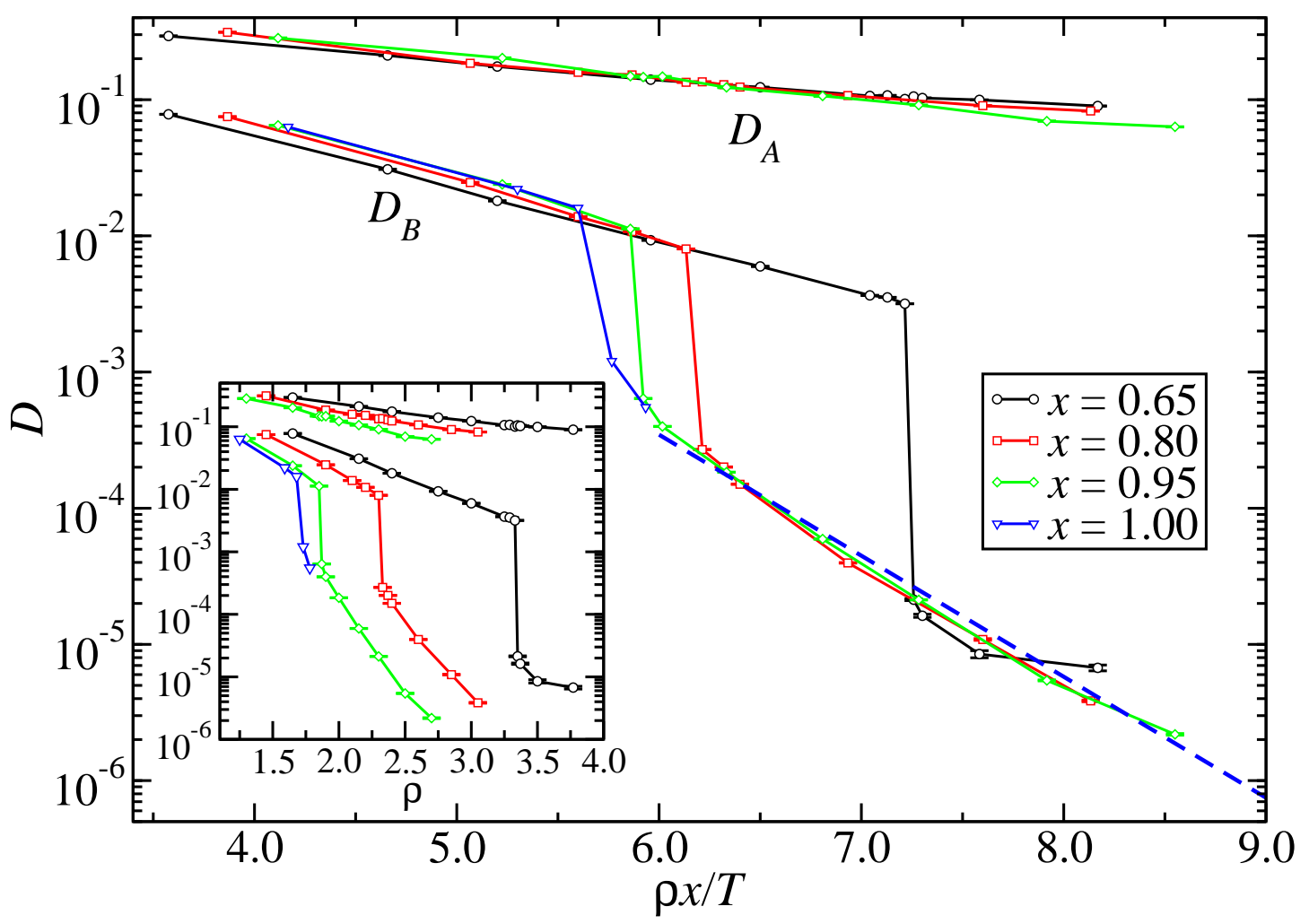

Figure 5. Diffusivities of the $A$ - and $B$-particles as a function of the density (inset) and of the combined variable $\rho x / T$ (main panel). Solid lines are guides for the eyes. The dashed line in the main panel indicates approximate Arrhenius behavior $D_{B} \sim \exp \left[-2.3 \rho_{B} / T\right]$ (see text).

$\lim _{t \rightarrow \infty}\left\langle\Delta r_{\alpha}^{2}(t)\right\rangle / 6 t$. Figure 5 shows the obtained results as a function of the quantity 
$\rho_{B} / T=\rho x / T$. For each composition $x$, an abrupt drop in the diffusivity of the $B$ particles is observed in a narrow range of density. This drop reflects the transition of the species $B$ from the fluid to the cluster crystal, and it is indeed expected from the previous observations in the MSD (see Figure 3). The approximate scaling behavior for $D_{B}$ in the cluster crystal phase generalizes the observation for the pure GEM-8 system [12, 13]. The latter was rationalized as the result of activated hopping motion between minima of the local potential energy, which are placed at the lattice sites [12, 13, 19, 20]. It was found that the separating energy barrier between neighboring sites scales as $\Delta U \approx 2.3 \rho_{B}$, leading to Arrhenius behavior $D_{B} \sim \exp [-\Delta U / T]$ [12, 13]. Data of Figure 5 show that this observation is not altered in the mixture over a broad range of values of $\rho x / T$. The introduction of the non-clustering $A$-particles does not even change significantly the former activation energy $\Delta U$.

\subsection{Scattering functions}

Relaxation of density fluctuations of wave vector $q$ are evaluated by means of the intermediate coherent and incoherent scattering functions. The coherent function is defined as

$$
F_{\alpha \beta}(q, t)=\frac{\left\langle\rho_{\alpha}(\mathbf{q}, t) \rho_{\beta}(-\mathbf{q}, 0)\right\rangle}{\left\langle\rho_{\alpha}(\mathbf{q}, 0) \rho_{\beta}(-\mathbf{q}, 0)\right\rangle}
$$

and characterizes collective $\alpha-\beta$ correlations, whereas the incoherent function accounts for self-correlations and is given by

$$
F_{\alpha}^{(\mathrm{s})}(q, t)=\frac{1}{N_{\alpha}}\left\langle\sum_{j=1}^{N_{\alpha}} \exp \left\{i \mathbf{q} \cdot\left[\mathbf{r}_{\alpha, j}(t)-\mathbf{r}_{\alpha, j}(0)\right]\right\}\right\rangle .
$$

With the used normalizations $F_{\alpha \beta}(q, 0)=F_{\alpha}^{(s)}(q, 0)=1$. Figure 6 shows results of the scattering functions for composition $x=0.95$ at densities above and below the crystallization line. The selected value of $q=4.0$ is not a reciprocal lattice vector (RLV) and therefore data in this figure reflect relaxation of out-of-lattice correlations. Consistently with the observations in the real space (see above), both incoherent and coherent functions for the species $A$ exhibit a fast decay at all the investigated densities, also for those where the $B$-particles form the cluster crystal. This is reminiscent of the scenario presented for the small particles in Yukawa mixtures immediately below the crystallization temperature [21]. In close similarity to the aforementioned work, our data reveal, for the crystalline phase, a weak oscillation at $t \sim 2$, immediately after the microscopic decay. It reflects short-time vibrational dynamics of the $A$-particles within the interstitials. This effect was not detected in the respective MSD (see above), possibly because contrary to the scattering functions, the former is generally dominated by fast contributions, which may mask the oscillations. As discussed above, these short-time oscillations and the similar ones observed for the scattering functions of the $B$-particles (Figures 6 and 7) are artifacts of the Newtonian dynamics and will be strongly damped or even vanish for $\mathrm{BD}$ and $\mathrm{MC}$ simulations. 

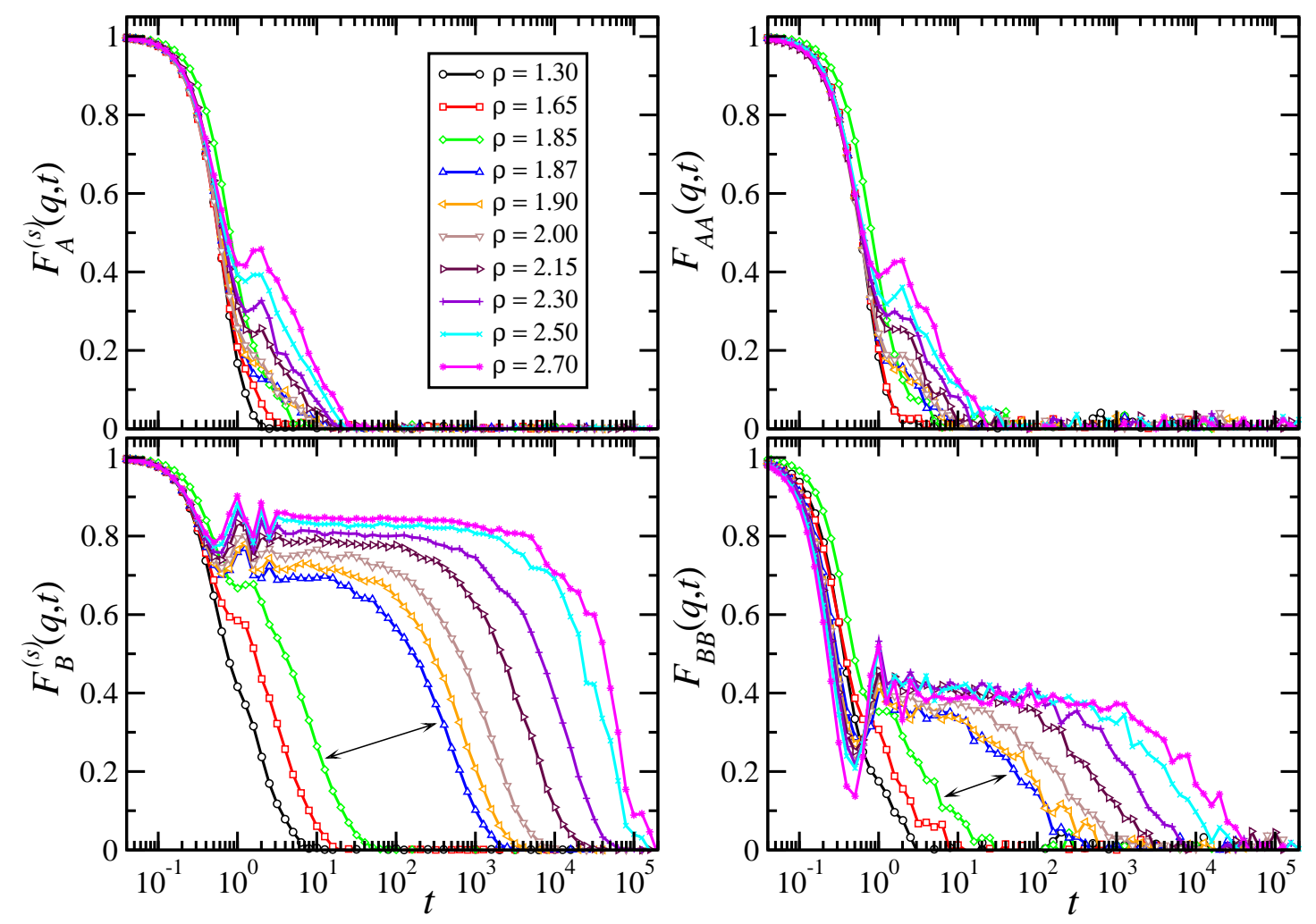

Figure 6. Intermediate incoherent and coherent scattering functions for fixed $x=0.95$ and $q=4.0$, at different densities (see legend for $F_{A}^{(\mathrm{s})}(q, t)$ ). The arrows in bottom panels separate fluid states for the $B$-particles from crystal clusters (see also Figure 1).

Concerning the dynamics of the clustering $B$-particles, the present system exhibits more complex features than usual crystals. As in real-space quantities (see above), coherent and incoherent functions reflect an abrupt slowing down of the dynamics by crossing the crystallization point. The intermediate plateau regime extends over longer time intervals as density increases, before the ultimate relaxation at long times. This observation is reminiscent of the usual dynamic scenario associated to the glass transition. Having noted this, distinct features are revealed by inspection of incoherent and coherent functions. Thus, the relaxation of the coherent function $F_{B B}(q, t)$ in the cluster crystal phase is consistent within statistics with a same plateau height (DebyeWaller factor, $f_{q}$ ) for all the investigated densities. This is not the case for the incoherent function $F_{B}^{(\mathrm{s})}(q, t)$, where the plateau height (Lamb-Mössbauer factor, $f_{q}^{(\mathrm{s})}$ ) clearly grows up as density increases.

The usual observation in glass-forming systems is that both Debye-Waller and Lamb-Mössbauer factors remain constant on approaching the glass transition from the ergodic phase. On crossing the transition and moving deep into the glassy phase, the former factors $f_{q}$ and $f_{q}^{(\mathrm{s})}$ progressively increase. This feature reflects the progressive decrease of the localization length respectively associated to collective and self-motions. With these ideas in mind, the increase of the plateau height in the incoherent functions 
for the $B$-particles (Figure 6) suggests that a localization transition has occurred for the self-motions, tentatively at the crystallization density. On the contrary, such a transition is not detected for out-of-lattice collective correlations at any of the investigated crystalline states. This dynamic decoupling, in the meaning of a different locus for the localization transitions of self- and out-of-lattice collective motions, is also present in the pure cluster crystal [12, 13. Having noted this, the ergodicity of self-motions is, as in the pure GEM-8 system, restored at long times. This is reflected in the full decay of $F_{\mathrm{B}}^{(\mathrm{s})}(q, t)$. In other words, the localization transition for self-motions of the $B$-particles is actually avoided. The mechanism which restores ergodicity of selfmotions is naturally the incessant hopping between neighboring clusters discussed above. Qualitatively similar findings are observed for the other studied compositions $x=0.8$ and $x=0.65$ (not shown). Thus, the addition of non-clustering $A$-particles, even up to a $35 \%$ of the total, does not alter the former scenario of dynamic decoupling in the $B$-particles, and the (avoided by hopping) localization transition for self-motions still occurs at much lower densities that for out-of-lattice collective correlations $\S$

As discussed in Refs. [12, 13], the former scenario has similarities and differences with the dynamics in plastic crystals, where molecules are constrained to vibrate around lattice sites, but perform full rotations which relax out-of-lattice collective correlations. In the case of cluster crystals, cluster deformation constitutes an additional mechanism of relaxation for such correlations. However, contrary to the case of plastic crystals, selfmotions can explore arbitrarily long distances in cluster crystals by means of activated hopping. This leads to non-zero diffusivities (Figure 5) and full relaxation of incoherent scattering functions (Figure 6).

As discussed above, the data presented in Figure 6 for a selected non-RLV $q=4.0$ display similar features, in the case of the species $B$, to those of the pure GEM- 8 system. Novel features are revealed for both species when the scattering functions are represented as a function of $q$. This is demonstrated in Figure 7 for a fixed crystalline state point, $x=0.95, \rho=2.70$, and for different values of $q$, including RLVs and nonRLVs. In the case of the $A$-particles, the incoherent function displays no signatures of glassy dynamics, but a fast decay for all the wave vectors. This is also the case for the coherent function at non-RLVs. Indeed the respective time scales are very similar for common $q$. However, a strong decoupling between self- and collective dynamics is observed for RLVs. Contrary to the incoherent case, the coherent function exhibits a plateau over several decades before relaxation at long times.

This dynamic decoupling is rather different from that above discussed for the species $B$ at non-RLVs. On the contrary, it is somewhat reminiscent of the scenario displayed by the small particles in disordered binary mixtures for certain ranges of composition and size ratio [22, 23]. In such systems the small particles move along a channel-like structure, formed by the interstitials of a matrix of large particles which relaxes in a much longer time scale, or which is even in the glassy state. As a consequence of the

$\S$ It should be mentioned, however, that although the $A$-particles constitute $35 \%$ of the number density of the system, they only account for less that $1 \%$ of its volume fraction due to their much smaller size. 

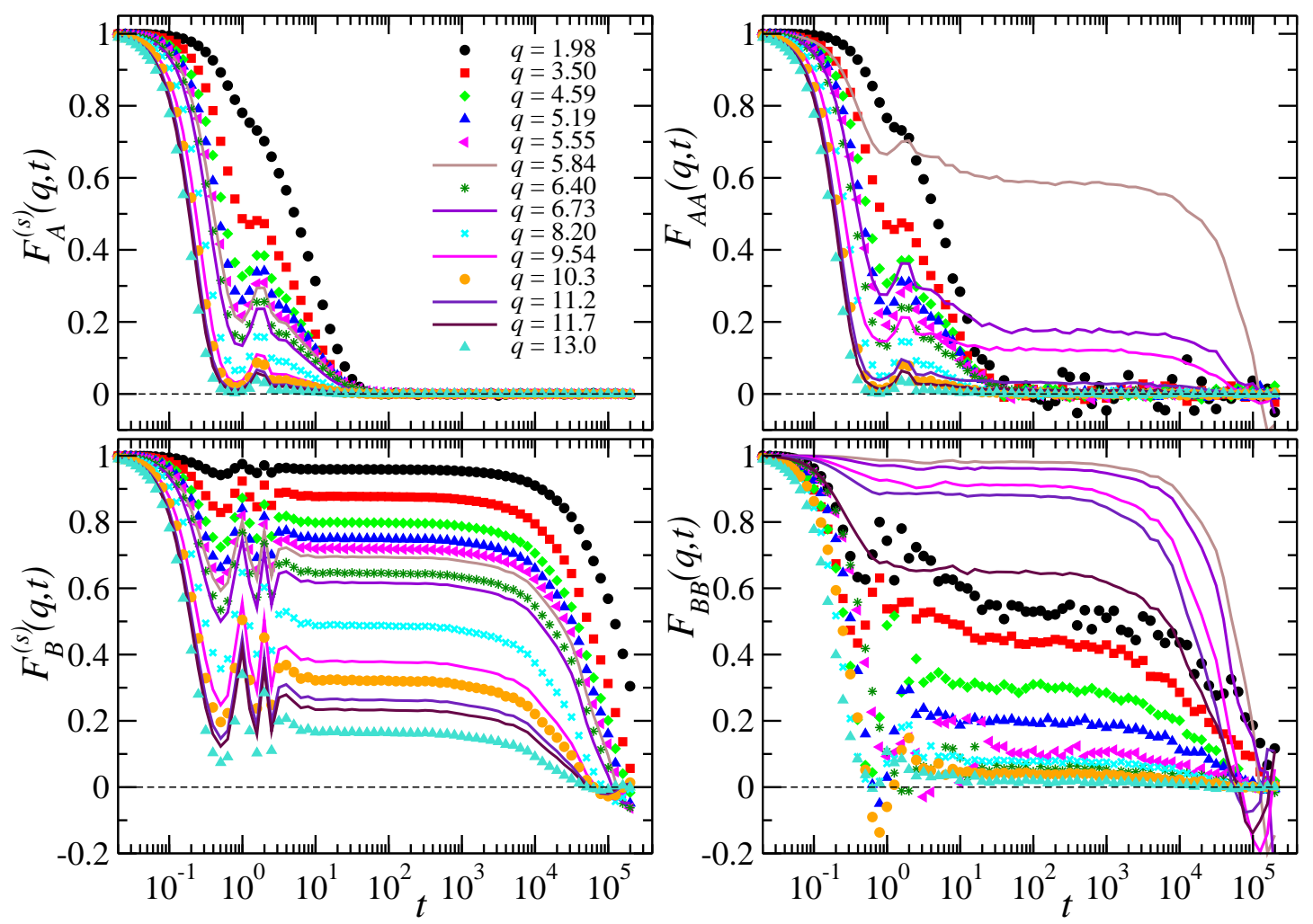

Figure 7. Intermediate incoherent and coherent scattering functions for fixed $x=0.95$ and $\rho=2.70$, at different wave vectors (see legend for $F_{A}^{(\mathrm{s})}(q, t)$ ). Solid lines and symbols correspond respectively to RLVs and to non-RLVs. The dashed lines indicate the zero value of the correlators.

quasistatic arrangement of the interstitials, collective correlations of the small particles decay very slowly, despite fast self-motions are performed, allowing for the exploration of large distances. Thus, decoupling of incoherent and coherent scattering functions of the small particles is observed in all the range of low and moderate wave vectors which probe the matrix structure [22, 23]. As discussed above, for the species $A$ of the mixtures here investigated, the former decoupling is only observed for RLVs. This reflects the fact that the non-clustering particles are preferentially located at the interstitials between the $B$-clusters, as was seen in the static correlation functions (Figure 2), and therefore also move preferentially between them. Since collective correlations between interstitial positions are probed by the RLVs, a decoupling between incoherent and coherent dynamics is observed for such wave vectors, in analogy with the scenario discussed above for disordered mixtures.

Concerning the scattering functions of the clustering species $B$, slow relaxation is observed for the incoherent and coherent cases, and both for RLVs and non-RLVs. As usual, Lamb-Mössbauer factors show a monotonic q-dependence, while Debye-Waller factors follow the modulations of the structure factor. Having noted this, coherent functions reveal non-trivial differences between RLVs and non-RLVs at the longest times of the simulation window. Namely, $F_{B B}(q, t)$ exhibits, for RLVs, an oscillation around 


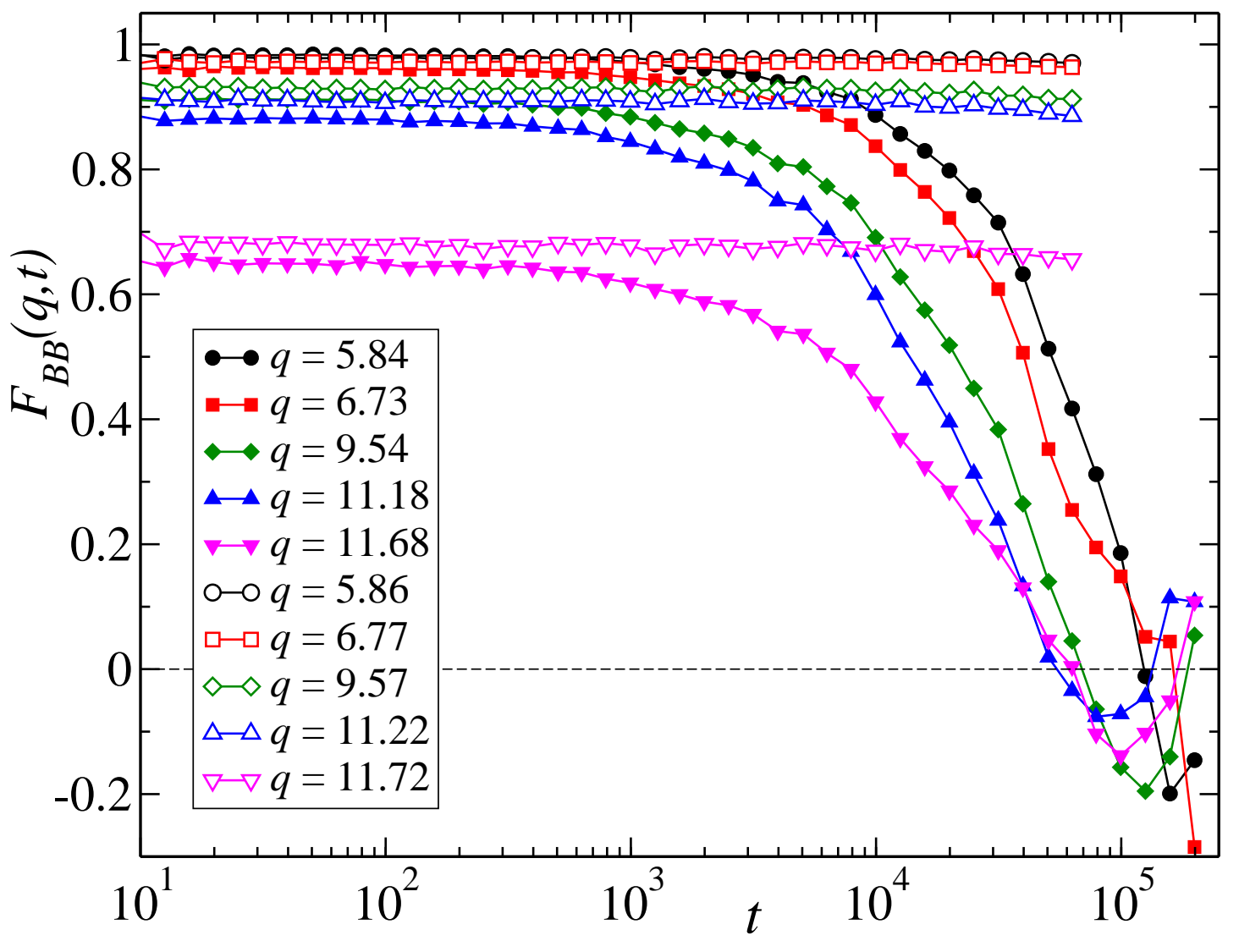

Figure 8. Intermediate coherent scattering functions of $B$-particles for several RLVs (see legend). Filled symbols correspond to the mixture with $x=0.95, \rho=2.70$, and $T=0.30$. Empty symbols correspond to the pure GEM- 8 system with $\rho=3.0$ and $T=0.40$. Solid lines are guides for the eyes. The dashed line indicates the level $F_{B B}(q, t)=0$.

$t \sim 10^{5}$. This oscillation is apparently absent for non-RLVs.

For the sake of clarity, the former data for the RLVs are shown separately in Figure 8 over a time window $t>10$. Given the large amplitude of the long-time anticorrelations, reaching values of $F_{B B}(q, t) \cong-0.2$ at the minimum, it is improbable that the oscillation around $t \sim 10^{5}$ is an statistical artifact. The figure also includes coherent data, at its respective RLVs, of the pure GEM-8 system at $\rho=3.0$ and $T=0.40$. The rather different behavior in the pure system and in the mixture, even with only a $5 \%$ of $A$ particles in the latter, becomes evident. While full relaxation followed by oscillation is observed in the mixture, no decay is present in the pure cluster crystal. It must be stressed that this difference is not related to a much slower intrinsic dynamics of the pure system for the selected state point $\rho=3.0$ and $T=0.40$. Indeed, by inspection of all the other dynamic observables introduced above, including coherent functions for non- $R L V s$, it is found that the pure system shows a faster dynamics than the mixture at the former state points. For example $D_{B} \approx 2 \times 10^{-6}$ and $10^{-5}$ respectively for the mixture and the pure system. Therefore, the oscillatory feature observed at long times 
in the mixture is induced by the addition of the gaussian $A$-particles.

The presence of negative correlations in the collective dynamics of $B$-particles at near RLV-wavevectors, reached at typical times $\tau_{a} \sim 10^{5}$, is a particular feature of the mixture, which is is absent for the pure $B$ system within the simulated time window. Physically, it describes a process by which a positive density correlation at $t=0$ at a distance of the order of the lattice constant turns negative at time scales $\tau_{a}$. As such, it points to the existence of some characteristic oscillation frequency $\omega_{a} \sim \tau_{a}^{-1}$, which corresponds to wavevectors lying at the edge of the Brillouin zone, i.e., a shortwavelength acoustical mode. Although such a process has not been seen for the pure $B$-system, phonon excitations exist for the pure system as well; it is thus reasonable to assume that the corresponding frequency for the pure system is much smaller that $\omega_{a}$, so that the time required for this relaxation process to be seen in the coherent scattering intensities is much longer. A detailed calculation of the phonon spectra of the mixture at hand, along parallel lines to the one recently carried out for pure systems [24] would shed light into this question, it is however cumbersome and it will be left as the subject of a future publication. Nevertheless, in view of the fact that the $A$-particles provide additional repulsions to the $B$-species and thus enhance the restoring forces that act on the latter, it appears plausible that in the mixture the phonon frequencies are larger than those in the pure system and thus the characteristic time $\tau_{a}$ becomes visible within the simulation window. Alternatively, the issue could also be resolved by performing very long simulations for the pure $B$-system.

\section{Conclusions}

The dynamic aspects of binary mixtures of ultrasoft (GEM-8 and gaussian) particles have been studied by means of MD. The present work extends previous results for the cluster crystal phase of the pure GEM-8 system, and investigates the effects of the addition of the non-clustering gaussian particles on the corresponding dynamic scenario. The obtained results show that as the total density increases at fixed composition, the GEM-8 species builds cluster crystals with a relatively high localization. As in the onecomponent system, the incoherent scattering functions indicate a localization transition for self-motions of the GEM-8 particles, which is avoided by incessant hopping motion between clusters. This transition seems to occur at lower densities than for out-of-lattice

collective correlations, confirming in the mixture the dynamic decoupling observed in the one-component cluster crystal.

On the other hand, the gaussian particles remain delocalized in the periodic potential induced by the GEM-8 clusters and display fast self-motions. However, slow collective dynamics is observed for specific wave vectors, namely those belonging to the reciprocal lattice. This feature reflects the preferential motion of the gaussian particles over the interstitials, confirming the expectations from static correlations. A striking feature is revealed by the analysis of collective correlations of the GEM-8 particles, for wave vectors at the reciprocal lattice, a feature which we attribute to the stiffening of 
an acoustical mode at the edge of the Brillouin zone, caused by the presence of the $A$ particles. An additional open question that should be the subject of future investigations is the influence of the type of elementary processes employed to model the dynamics in the mixture, along the lines of the work by Coslovich et al. carried out for pure systems [18. Here, it should be examined which of the features discovered by means of our MD approach survive if one employs Monte Carlo moves instead, which are expected to mimic better the overdamped, Brownian Dynamics of the system.

Finally, while there is evidence that both classes of ultrasoft potentials accurately model the interactions between real macromolecules in dilute systems, the validity of these potentials in dense systems has yet to be verified. The results presented in this paper add to a growing body of work which suggests that, the search for dense systems where ultrasoft potentials remain valid is a worthwhile pursuit.

\section{Acknowledgments}

This work has been supported by the projects NMP3-CT-2004-502235 (Network of Excellence SoftComp, EU) and ITN-234810-COMPLOIDS (Marie Curie Training Network, EU). M.C. acknowledges a PhD fellowship from the German Academic Exchange Service (DAAD, Germany) and the ALECOL Program (Colombia), as well as support from Donostia International Physics Center (Spain).

\section{References}

[1] Louis A A, Bolhuis P G, Hansen J P, and Meijer E J, 2000 Phys. Rev. Lett. 852522

[2] Bolhuis P G, Louis A A, Hansen J P, and Meijer E J, 2001 J. Chem. Phys. 1144296

[3] Likos C N, Rosenfeldt S, Dingenouts N, Ballauff M, Lindner P, Werner N and Vögtle F, $2002 \mathrm{~J}$. Chem. Phys. 1171869

[4] Likos C N, Lang A, Watzlawek M and Löwen H, 2001 Phys. Rev. E 63031206

[5] Glaser M A, Grason G M, Kamien R D, Košmrlj A, Santangelo C D and Zieherl P, 2007 Eur. Phys. Lett. 7846004

[6] Mladek B M, Kahl G and Likos C N, 2008 Phys. Rev. Lett. 100028301

[7] Lenz D A, Blaak R, and Likos C N, 2009 Soft Matter 52905

[8] Narros A, Moreno A and Likos C N, 2010 Soft Matter 62435

[9] Likos C N, Mladek B M, Gottwald D and Kahl G, 2007 J. Chem. Phys. 126224502

[10] Mladek B M, Kahl G and Likos C N, 2007 J. Chem. Phys. 126224502

[11] Lang A, Likos C N, Watzlawek M, and Löwen H. 2000 J. Phys.: Condens. Matter 125087

[12] Moreno A J and Likos C N, 2007 Phys. Rev. Lett. 99107801

[13] Likos C N, Mladek B M, Moreno A J, Gottwald D, and Kahl G, 2008 Comput. Phys. Commun. 17971

[14] Overduin S and Likos C N, 2009 Eur. Phys. Lett. 8526003

[15] Overduin S and Likos C N, 2009 J. Chem. Phys. 131034902

[16] Hansen J P and McDonald I R, 2006 Theory of simple liquids (London, Academic Press)

[17] Frenkel D and Smit B, 1996 Understanding Molecular Simulation (San Diego, Academic Press)

[18] Coslovich D, Strauss L and Kahl G, arXiv:1006.4982

[19] This hopping scenario shows striking analogies, both for van Hove functions and potential energy profiles, with the layer-to-layer diffusion of rods in smectic phases (see Ref. [20]). In such systems the corresponding energy barriers separate minima located in the centers of the layers. The 
incoherent scattering functions of the rods and the $B$-particles (see section 3.3) also exhibit similar trends [20].

[20] Matena R, Dijkstra M and Patti A, 2010 Phys. Rev. E 81021704

[21] Kikuchi N and Horbach J, 2007 Eur. Phys. Lett. 7726001

[22] Moreno A J and Colmenero J, 2006 J. Chem. Phys. 125164507

[23] Voigtmann Th and Horbach J, 2009 Phys. Rev. Lett. 103205901

[24] Neuhaus T and Likos C N, arXiv:1008.1881 